PERSPECTIVE

\title{
An evaluation of sustainability and societal impact of high-power laser and fusion technologies: a case for a new European research infrastructure
}

\author{
S. Atzeni ${ }^{1}$, D. Batani ${ }^{2}$, C. N. Danson ${ }^{3,4}$, L. A. Gizzi ${ }^{5}$, M. Perlado ${ }^{6}$, M. Tatarakis ${ }^{7,8}$, V. Tikhonchuk ${ }^{2,9}$, \\ and L. Volpe ${ }^{10,11}$ \\ ${ }^{1}$ Dipartimento SBAI, Università di Roma "La Sapienza", Roma 00161, Italy \\ ${ }^{2}$ CELIA, Université de Bordeaux-CNRS-CEA, UMR 5107, 33405 Talence, France \\ ${ }^{3} A W E$, Aldermaston, Reading RG7 4PR, UK \\ ${ }^{4}$ Centre for Intertial Fusion Studies, Blackett Laboratory, Imperial College London, London SW7 2AZ, UK \\ ${ }^{5}$ Istituto Nazionale di Ottica, Consiglio Nazionale delle Ricerche, 56125 Pisa, Italy \\ ${ }^{6}$ Instituto Fusión Nuclear “Guillermo Velarde” Universidad Politecnica, 28006 Madrid, Spain \\ ${ }^{7}$ Institute of Plasma Physics and Lasers, Hellenic Mediterranean University Research Centre, 74100 Rethymno, Greece \\ ${ }^{8}$ Department of Electronic Engineering, Hellenic Mediterranean University, 73133 Chania, Greece \\ ${ }^{9}$ ELI-Beamlines Center, Institute of Physics, Czech Academy of Sciences, 25241 Dolni Brezany, Czech Republic \\ ${ }^{10}$ Centro de Laseres Pulsados (CLPU), Parque Cientifico, E-37185 Villamayor, Salamanca, Spain \\ ${ }^{11}$ Laser-Plasma Chair at the University of Salamanca, E-37185 Villamayor, Salamanca, Spain \\ (Received 10 September 2021; accepted 14 September 2021)
}

\begin{abstract}
Fusion energy research is delivering impressive new results emerging from different infrastructures and industrial devices evolving rapidly from ideas to proof-of-principle demonstration and aiming at the conceptual design of reactors for the production of electricity. A major milestone has recently been announced in laser fusion by the Lawrence Livermore National Laboratory and is giving new thrust to laser-fusion energy research worldwide. Here we discuss how these circumstances strongly suggest the need for a European intermediate-energy facility dedicated to the physics and technology of laser-fusion ignition, the physics of fusion materials and advanced technologies for high-repetitionrate, high-average-power broadband lasers. We believe that the participation of the broader scientific community and the increased engagement of industry, in partnership with research and academic institutions, make most timely the construction of this infrastructure of extreme scientific attractiveness.
\end{abstract}

Keywords: fusion energy; high power lasers; plasmas; inertial fusion; high energy density

Fusion energy research is currently pursuing different routes, leading to scientific and technological developments in different areas, with significant industrial impact generated mainly by the magnetic fusion approach. Major advancements have been demonstrated in vacuum technology, mechanical and control systems, cryogenics, numerical simulations and advanced materials, just to cite a few. This impact is fuelled by the significant institutional public funding that is supporting magnetic fusion energy

Correspondence to: L. A. Gizzi, Istituto Nazionale di Ottica, Consiglio Nazionale delle Ricerche, 56125 Pisa, Italy. Email: la.gizzi@ino.cnr.it programmes worldwide and, in particular, the ITER and related test facilities, such as the JET in Oxfordshire (UK), DTT in Frascati (Italy) and JT-60SA in Naka (Japan).

At the same time, in the past two decades, a number of independent initiatives targeted at developing alternative fusion reactor concepts have been emerging and developing, funded by private investors, aimed at compact solutions in ambitiously rapid timeframes. These enterprises leverage the growing awareness of the forthcoming crisis of fossil fuels and related environmental issues, and the recent institutional guidelines driving the development of alternative and renewable energy supplies. These circumstances are 
attracting investors towards approaches that have an established scientific rationale, although yet to be established for energy production, like most of the schemes currently under investigation $^{[1]}$.

While magnetic fusion energy research has been around for almost 80 years, more recent approaches, such as the laser-driven fusion energy research, have been developing over the past 50 years and have recently achieved laboratory demonstration ${ }^{[2]}$ of ignition-like conditions, with fusion energy production largely exceeding absorbed energy and approaching the laser input energy (gain 1), a pre-requisite for the development of an experimental reactor. In the meantime, the scientific community traditionally dealing with the scientific building blocks of laser-fusion energy, namely high-power lasers, high-energy-density physics and laser-plasma interaction physics, has grown and diversified in several areas, giving rise to entirely new areas of research.

New installations based on high-energy laser systems (tens of $\mathrm{kJ}$ up to a few MJ) for inertial confinement fusion (ICF) and inertial fusion energy (IFE) research were conceived and built, such as Omega and the NIF in the United States, LMJ in France, Vulcan and Orion in the UK, SG-II and SG-III in China and Gekko XII in Japan. In parallel, the advent of chirped pulse amplification led to the construction of ultrashort pulse, ultra-high-intensity laser facilities, of many new medium and large-scale facilities at a national level and eventually led to the European Extreme Light Infrastructure, currently in the commissioning stage in the European Union (EU) at three different sites in the Czech Republic, Romania and Hungary. Construction of these installations has required rapid scientific and technological developments on a very short time scale aimed at high-field science, particle acceleration and secondary radiation sources (see Figure 1). Many of these developments have also generated industrial products that are also impacting on other commercial areas including, for example, the medical and manufacturing industries.

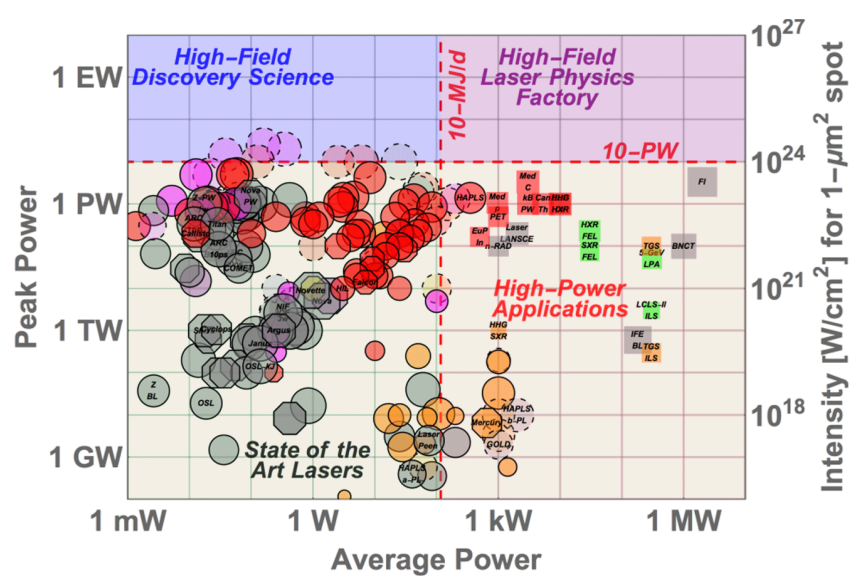

Figure 1. A map of current lasers' peak power versus average power ${ }^{[3]}$.
The scientific communities in high energy density, plasma physics and high-power laser technology are working closely with each other, with large European research institutes having active research programmes in all of these areas, which share common background knowledge. The laserplasma community is overall a large community that is now further expanding and is also partially merging with the synchrotron and X-ray free electron laser (FEL) communities, a sharing of common interest in investigating extreme states of matter with ultrashort and high-brightness $\mathrm{X}$-ray pulses. These communities also share advanced particle and radiation diagnostics and high-power laser technologies.

It is clear that third and fourth generation light sources on the one hand and high-power, high-intensity laser sources, on the other, are at the core of many of the latest technological and industrial developments. However, access to these large facilities is still strongly limited due to their high cost and large size. A further revolution is needed to enable them to shrink in size, to reduce the cost and become affordable, potentially table top, industrial tools. In this context, high-power solid-state lasers have been traditionally limited in average power and repetition rate by the use of the well-established flashlamp pumping approach and related thermal issues that have undermined high-power lasers since their birth. New approaches based on highefficiency diode pumping are developing fast, paving the way to high-average-power and high-repetition-rate lasers. This transition is going to likely make lasers and laser-based light sources available to a broader community, empowering small and medium high-tech enterprises, making them capable of industrial research currently only accessible at large installations.

The transition of high-power lasers to high average power and high repetition rate is also a key milestone of laserfusion energy research. It is required for the transition from proof-of-principle demonstration of ignition of fusion reactions to repetitive operation required in a future reactor for continuous energy production. Technical specifications for laser-fusion energy are extremely challenging, requiring megajoule energy in nanosecond pulses at ultraviolet (UV) wavelength, but the core of the technology is largely overlapping, with other industrial applications requiring innovative and ground-breaking solutions.

Several laser companies are currently investing significant resources in developing substantially new high-averagepower laser technology, delivering new products that include solid-state diode laser pumping. This will also contribute to the demand for high-power diode laser technology, leading to more efficient and cost-effective production.

In this scenario, laser-fusion energy research may play a driving role, setting additional demanding laser specifications that will require further developments. As anticipated above, these developments are also shared to a great extent 
with other emerging technologies, including laser-plasma accelerators for medical applications and non-destructive material testing, that require high-average-power sources. In this context, broadband, high-power laser technology is rapidly emerging as a common enabling block, which is essential for commercial applications of ultra-intense lasers and may also be required in laser-driven fusion to overcome outstanding issues in controlling laser-plasma interactions. Broadband high-power lasers are essential, for example, to enable high-repetition-rate capabilities for laser-driven charged particle accelerators or to ensure smooth and uniform energy delivery in IFE. Such high-power broadband lasers require significant innovation, such as new lasing materials capable of being pumped by commercial diode lasers and with advanced mechanical and thermal properties, such as ceramic materials.

Significant progress in the multidisciplinary science of materials at extreme conditions has enabled great improvement of the laser-fusion chamber design, which is the key part of the IFE reactor. This includes a better understanding of materials under extreme irradiation conditions, tritium retention assessment and thermo-fluid dynamics for the cooling and energy recovering systems.

With all these advances in place, laser-fusion energy research could act as an incubator, driving the industrial development of components in all these areas, aiming at an entirely new approach, potentially including innovative compact solutions for a future power plant. On this path, the latest achievements in laser fusion at the $\mathrm{NIF}^{[5]}$, notably the $1.3 \mathrm{MJ}$ of fusion energy output and the $230 \mathrm{~kJ}$ of kinetic energy of the imploding shell from 1.9 MJ total laser energy input, should be regarded as a major milestone that places ICF as a valuable candidate among the very few approaches capable of ignition and controlled fusion. The efficiency of the ignition scheme tested at the NIF is inherently capped by the 'indirect drive approach', where laser energy is used inefficiently to heat the hohlraum and generate the X-ray radiation required for the fuel compression.

The 'direct drive' approach, including 'shock ignition ${ }^{\mathrm{S}]}$, promises a much more efficient use of laser energy to directly illuminate the fuel capsule and drive its compression. In the 'shock ignition' approach, in particular, a temporally shaped laser pulse is used, leading to a moderate compression phase followed by an intense, shock driving pulse that leads to the activation of a convergent shock capable of triggering ignition of the pre-compressed fuel. Shock ignition has been investigated now for more than a decade at a fundamental level, to unfold issues related to the strong non-linear interaction $^{[7,8]}$ of the shock driving pulse. This concept was at the core of the HiPER project (European High Power laser Energy Research facility ${ }^{[4]}$ and is currently being considered by the academic community for future proof-of-principle demonstration at the LMJ or at the NIF.

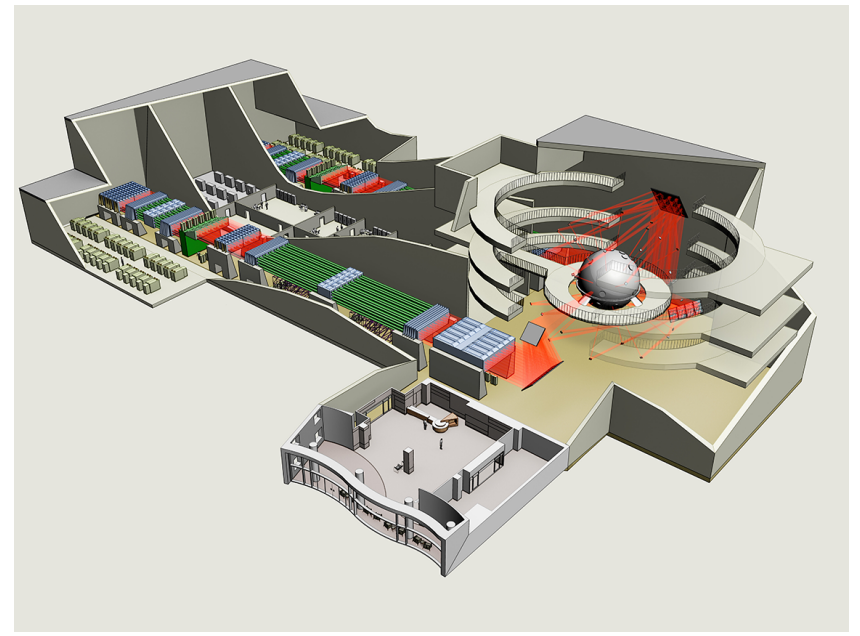

Figure 2. Original concept of the HiPER facility for demonstration of direct-drive laser fusion ${ }^{[4]}$.

Indeed, a strong laser-fusion scientific community exists in Europe, which is actively pursuing all opportunities for shock ignition demonstration at a new dedicated facility (see Figure 2), in national laboratories and also in the framework of the collaborative European project on 'Advancing shock ignition for direct-drive inertial fusion', a unique laser-fusion Enabling Research Project funded by EUROFUSION, the European consortium on fusion energy. The high societal impact, the strong scientific background and attractiveness for industrial development of laser-fusion energy research could make this facility a unique research infrastructure and, most likely, a launch pad for future IFE.

\section{References}

1. Fusion news ignites optimism. Nat. Photon. 15, 713 (2021).

2. https://www.llnl.gov/news/national-ignition-facilityexperiment-puts-researchers-threshold-fusion-ignition.

3. C. N. Danson, C. Haefner, J. Bromage, T. Butcher, J.-C. F. Chanteloup, E. A. Chowdhury, A. Galvanauskas, L. A. Gizzi, J. Hein, D. I. Hillier, N. W. Hopps, Y. Kato, E. A. Khazanov, R. Kodama, G. Korn, R. Li, Y. Li, J. Limpert, J. Ma, C. H. Nam, D. Neely, D. Papadopoulos, R. R. Penman, L. Qian, J. J. Rocca, A. A. Shaykin, C. W. Siders, C. Spindloe, S. Szatmári, R. M. G. M. Trines, J. Zhu, P. Zhu, and J. D. Zuegel, High Power Laser Sci. Eng. 7, e54 (2019).

4. http://www.hiper-laser.org/.

5. D. Clery, Science 373, 841 (2021).

6. R. Betti, C. D. Zhou, K. S. Anderson, L. J. Perkins, W. Theobald, and A. A. Solodov, Phys. Rev. Lett. 98, 155001 (2007).

7. D. Batani, L. Antonelli, F. Barbato, G. Boutoux, A. Colaïtis, J.-L. Feugeas, G. Folpini, D. Mancelli, Ph. Nicolai, J. Santos, J. Trela, V. Tikhonchuk, J. Badziak, T. Chodukowski, K. Jakubowska, Z. Kalinowska, T. Pisarczyk, M. Rosinski, M. Sawicka, F. Baffigi, G. Cristoforetti, F. D'Amato, P. Koester, L. A. Gizzi, S. Viciani, S. Atzeni, A. Schiavi, M. Skoric, S. Gus'kov, J. Honrubia, J. Limpouch, O. Klimo, J. Skala, Y. J. 
Gu, E. Krousky, O. Renner, M. Smid, S. Weber, R. Dudzak, M. Krus, and J. Ullschmied, Nuclear Fusion 59, 032012 (2019).

8. G. Cristoforetti, L. Antonelli, D. Mancelli, S. Atzeni, F. Baffigi, F. Barbato, D. Batani, G. Boutoux, F. D'Amato, J.
Dostal, R. Dudzak, E. Filippov, Y. J. Gu, L. Juha, O. Klimo, M. Krus, S. Malko, A. S. Martynenko, Ph. Nicolai, V. Ospina, S. Pikuz, O. Renner, J. Santos, V. T. Tikhonchuk, J. Trela, S. Viciani, L. Volpe, S. Weber, and L. A. Gizzi, High Power Laser Sci. Eng. 7, e51 (2019). 